\title{
The existence of contingent epiderivative for a set-valued mapping and vector variational-like inequalities
}

\author{
Yanfei Chai ${ }^{* *}$, Sanyang Liu and Yanying $\mathrm{Li}^{1,2}$
}

\section{"Correspondence:}

chyf_0923@163.com

'Department of Mathematics,

Xidian University, Xi'an, 710071,

China

Full list of author information is

available at the end of the article

\begin{abstract}
In this paper we analyze the existence of the contingent epiderivative for a set-valued mapping in a general normed space with respect to Daniell cone. As its application, we will also establish the relationships between set-valued optimization problems and variational-like inequality problems under the conditions of pseudo invexity. MSC: 49J52; 90C56
\end{abstract}

Keywords: set-valued optimization; ideal minimal points; contingent epiderivative; existence conditions; strong variational-like inequalities

\section{Introduction}

In [1], Jahn and Raüh introduced the notion of contingent epiderivative for a set-valued mapping, which modifies a notion introduced by Aubin [2] as upper contingent derivative, and also established the existence theory of this contingent epiderivative for a singlevalued function. In [3], Jahn and Khan obtained the existence of this kind of contingent epiderivative for a real set-valued function. It has been shown that this notion of contingent epiderivative is a fundamental concept for the formulation of optimality conditions in set-valued optimization, but there are few works that study its existence for a set-valued mapping in general conditions. Although in [4] Rodríguez-Marín and Sama derived the existence of contingent epiderivative, this can only be assured if a set-valued mapping $F$ has the $L B D$ (lower bounded derivative) property. In the last decades, many researchers have given several other generalized notions of epiderivatives by using weak minimizers and minimizers and derived the existence theories for them; see [5] and [4, 6, 7], respectively. Using different kinds of minimal elements, one can define different kinds of epiderivatives. In our paper, we use the ideal minimal elements of a set and the concept of contingent cone to define the contingent epiderivative and analyze its domain, existence, uniqueness and other properties. Under determined conditions, we establish $\operatorname{dom}\left(D F\left(x_{0}, y_{0}\right)\right)=\operatorname{cone}\left(A-x_{0}\right)=T\left(A, x_{0}\right)$ and get the existence of $D F\left(x_{0}, y_{0}\right)$, where $\operatorname{dom} F$ is the domain of $F, D F\left(x_{0}, y_{0}\right)$ is the contingent epiderivative of $F$ at $\left(x_{0}, y_{0}\right) \in \operatorname{gr} F$ (the graph of $F$ is denoted by $\operatorname{gr} F$ ) and $T\left(A, x_{0}\right)$ is the contingent cone of $A$ at $x_{0}$, respectively.

Our other purpose in this paper is to investigate the relationships between set-valued optimization problems and variational-like inequality problems. In fact, the relationships between vector variational-like inequality problems and optimization problems for a singlevalued mapping have been studied by many authors, see [8-10] and so on; and in [11], Zeng 
and $\mathrm{Li}$ also discussed the relationships between weak vector variational-like inequality problems and set-valued optimization problems. However, to the best of our knowledge, there are few papers discussing the solution relationships between set-valued optimization problems and strong vector variational-like inequality problems. Motivated by the works in [9] and [11], in this paper, we firstly introduce several kinds of generalized invexity for set-valued mappings and then prove that the solutions of the variational-like inequality problems are equivalent to the minima (ideal minima) of set-valued optimization problems.

The rest of the paper is organized as follows. In Section 2, we give some preliminaries and recall the main notions of contingent cone. In Section 3, the concept of contingent epiderivatives is introduced and, under determined conditions, its existence theory is also established. In Section 4, we present that the solutions of the variational-like inequality problems are equivalent to the minima (ideal minima) of set-valued optimization problems.

\section{Preliminaries and notations}

Throughout the paper, if not stated otherwise, let $X$ be a real normed space, let $A$ be a nonempty subset of $X$, and let $Y$ be a real normed space partially ordered by a closed, convex and pointed cone $D \subset Y$. The points of origin of all real normed spaces are denoted by $0_{X}$ and $0_{Y}$.

Let $y_{1}, y_{2} \in Y$, the orderings are defined in $Y$ as follows:

$$
\begin{aligned}
& y_{1} \not \leq y_{2} \Leftrightarrow y_{2}-y_{1} \notin D ; \\
& y_{1} \leq y_{2} \Leftrightarrow y_{2}-y_{1} \in D .
\end{aligned}
$$

Let $A \subset X, F: A \rightarrow 2^{Y}$ be a set-valued mapping. The graph, the epigraph and the domain of $F$ are defined, respectively, by

$$
\begin{aligned}
& \operatorname{gr} F=\{(x, y) \in X \times Y: y \in F(x)\}, \\
& \operatorname{epi} F=\{(x, y) \in X \times Y: y \in F(x)+D\},
\end{aligned}
$$

and

$$
\operatorname{dom} F=\{x \in X: F(x) \neq \emptyset\} .
$$

We say that $F$ is a $D$-convex set-valued mapping on $A$, if $A$ is a convex set, and for all $x, y \in A$, all $\lambda \in[0,1]$,

$$
\lambda F(x)+(1-\lambda) F(y) \subset F(\lambda x+(1-\lambda) y)+D .
$$

It is well known that if $F$ is $D$-convex on $A$, then epi $F$ is a convex subset in $X \times Y$.

Proposition 2.1 (see [12]) Let $F: X \rightarrow 2^{Y}$. If epi $F$ is a closed subset in $X \times Y$, then $F(x)+D$ is a closed set for each $x \in X$.

Definition 2.1 Let $B$ be a non-empty subset of $Y$.

(i) $y_{0} \in B$ is called a minimal point of $B$ with respect to cone $D$ if $\left(B-y_{0}\right) \cap-D=\left\{0_{Y}\right\}$;

(ii) $y_{0} \in B$ is called an ideal minimal point of $B$ with respect to cone $D$ if $B-y_{0} \subseteq D$. 
The elements of all minimal points and ideal minimal points of $B$ are denoted by $\operatorname{Min} B$ and $\operatorname{IMin} B$, respectively.

Obviously, IMin $B \subseteq \operatorname{Min} B$ if $D$ is a pointed cone.

The following standard notions can be found in [6].

Definition 2.2 Let $D$ be a closed, convex and pointed cone in $Y$.

(i) The cone $D$ is called Daniell cone if any decreasing sequence in $Y$ having a lower bound converges to its infimum.

(ii) A subset $B$ of $Y$ is said to be $D$-lower bounded (or be minorized) if there is an element $y \in Y$ such that $B \subset y+D$.

Proposition 2.2 Let D be a closed, convex and pointed cone in $Y$, non-empty subset $B \subset Y$. If $\operatorname{Min} B \neq \emptyset$ and $B$ is the $D$-lower bounded for every minimal point, i.e.,

for each $\bar{y} \in \operatorname{Min} B, \quad B \subset \bar{y}+D$,

then $\operatorname{Min} B$ is a single-point set and $\operatorname{Min} B=\operatorname{IMin} B$.

Proof We prove this result by contradiction. Suppose that at least there are $y_{1}, y_{2} \in \operatorname{Min} B$ and $y_{1} \neq y_{2}$. By condition (2.1), we have

$$
y_{1} \in y_{2}+D
$$

and

$$
y_{2} \in y_{1}+D \text {. }
$$

From (2.2), (2.3) and the assumption $y_{1} \neq y_{2}$, one has $y_{1}-y_{2} \in D \backslash\left\{0_{Y}\right\}$ and $y_{2}-y_{1} \in D \backslash\left\{0_{Y}\right\}$, which contradicts $D$ being a pointed cone, thus $y_{1}=y_{2}=\bar{y}$. Namely, Min $B$ is a singleton. Again from (2.1), we have

$$
B \subset \bar{y}+D
$$

i.e.,

$$
B-\bar{y} \subset D,
$$

this yields $\bar{y} \in \operatorname{IMin} B$. The proof is complete.

Remark 2.1 We notice if $\operatorname{Min} B \neq \emptyset$ and (2.1) is fulfilled, then IMin $B=\operatorname{Min} B \neq \emptyset$ is a singlepoint set. Clearly, if $\operatorname{IMin} B \neq \emptyset$, then $B \subseteq \operatorname{IMin} B+D$.

The following theorem is due to Borwein.

Theorem 2.1 (see [13]) Assume that D is a pointed, convex and Daniell cone, and let $B$ be a closed subset of $Y$. If $B$ is D-lower bounded (or is minorized), then $\operatorname{Min} B \neq \emptyset$. 
Definition 2.3 For $A \subset X$, $\operatorname{cone} A$ and $\operatorname{cl} A$ denote its cone hull and closure of $A$, respectively.

Furthermore,

$$
\text { cone } A=\{\lambda a: \lambda \geq 0, a \in A\} .
$$

Let $A \subset X$, the contingent cone of $A$ at $x_{0}$ is defined by

$$
T\left(A, x_{0}\right)=\left\{u \in X: \exists t_{n} \rightarrow 0^{+}, \exists u_{n} \rightarrow u, \exists n_{0} \in N, \forall n \geq n_{0}, x_{0}+t_{n} u_{n} \in A\right\} .
$$

It is well known that if $A$ is a convex set and $x_{0} \in A$, then

$$
T\left(A, x_{0}\right)=\operatorname{clcone}\left(A-x_{0}\right) .
$$

We say that $A \subset X$ satisfies the property $\digamma$, if for any $x \in A$ and $\lambda \in[0,1]$, one has $\lambda x \in A$. Let $0_{X} \in A$, we say that $A$ satisfies the property $\Lambda$ near the $0_{X}$, if there exists neighborhood $B\left(0_{X}, \varepsilon\right)$ such that for any $x \in B\left(0_{X}, \varepsilon\right) \cap(\operatorname{cl} A \backslash \operatorname{int} A)$ and $\lambda \in[0,1]$, one has $\lambda x \in(\operatorname{cl} A \backslash \operatorname{int} A)$, where $B\left(0_{X}, \varepsilon\right)$ denotes the ball centered at $0_{X}$ with radius $\varepsilon$.

Corollary 1 Let $A \subset X$ be a convex set, $x_{0} \in A$, then $A-x_{0}$ satisfies the property $\digamma$.

Proof For any $x \in A$ and $t \in[0,1], t\left(x-x_{0}\right)+x_{0}=t x+(1-t) x_{0}$, thereby, $t\left(x-x_{0}\right)+x_{0} \in A$ follows immediately from $A$ being a convex set, which implies $t\left(x-x_{0}\right) \in A-x_{0}$, the proof is complete.

Proposition 2.3 Let $A \subset X$, if $A$ satisfies the properties $\digamma$ and $\Lambda$ near the $0_{X}$, then $\operatorname{cone}(\operatorname{cl} A)=\operatorname{cl}(\operatorname{cone} A)$.

Proof Obviously, $0_{X} \in \operatorname{cone}(\operatorname{cl} A)$ and $0_{X} \in \operatorname{cl}(\operatorname{cone} A)$, so we consider $u \neq 0_{X}$ in the sequel. First, we prove the inclusion $\operatorname{cone}(\operatorname{cl} A) \subseteq \operatorname{cl}(\operatorname{cone} A)$. Let $u \in \operatorname{cone}(\operatorname{cl} A)$, then there exist $a \in \operatorname{cl} A, t>0$ such that $u=t a$. Furthermore, following $a \in \operatorname{cl} A$, there exists $\left\{a_{n}\right\} \subset A$, $a_{n} \rightarrow a$. For each $n$, set $u_{n}=t a_{n}$, then $u_{n} \in \operatorname{cone} A$ and $u_{n}=t a_{n} \rightarrow t a=u$, i.e., $u_{n} \rightarrow u$, this implies $u \in \operatorname{cl}(\operatorname{cone} A)$.

For the contrary inclusion, we should only prove cone $(\operatorname{cl} A)$ is a closed cone. Let $\left\{u_{n}\right\} \subset$ cone $(\operatorname{cl} A)$ and $u_{n} \rightarrow u$. Next, we will prove $u \in \operatorname{cone}(\operatorname{cl} A)$. In fact, we can conclude that there exist $x_{n} \in X$ and $x_{n} \rightarrow 0_{X}$ such that $u+x_{n}=u_{n} \in \operatorname{cone}(\operatorname{cl} A)$. So, for each $n$, there exist $t_{n}>0, a_{n} \in \operatorname{cl} A$ such that

$$
u+x_{n}=u_{n}=t_{n} a_{n} .
$$

On the one hand, if $a_{n} \rightarrow 0_{X}$, then $t_{n} \rightarrow+\infty$ as $n \rightarrow+\infty$. For each $n$, we have

$$
\frac{u}{t_{n}}+\frac{x_{n}}{t_{n}}=a_{n} \in \operatorname{cl} A
$$

For given $\varepsilon>0$, there exists $n_{0}$ such that $a_{n} \in B\left(0_{X}, \varepsilon\right), \forall n \geq n_{0}$. We divide it into two cases to discuss. 
(i) If $\left\{a_{n}\right\} \subset \operatorname{cl} A \backslash \operatorname{int} A$ as $n \geq n_{0}$, by assumption, we can conclude that there exists a subsequence $\left\{a_{n_{m}}\right\} \subseteq\left\{a_{n}\right\}$ such that

$$
a_{n_{m}}=\lambda_{n_{m}} a_{\bar{n}_{0}}
$$

with $\lambda_{n_{m}} \in(0,1]$ and $\bar{n}_{0} \geq n_{0}$. In fact, since $t_{n} a_{n} \rightarrow u$, so for any $\varepsilon_{1}>0$, there exists $n_{1} \geq n_{0}$ such that $\left\|t_{n} a_{n}-u\right\| \leq \varepsilon_{1}$ as $n \geq n_{1}$. If for any given $\bar{n}_{0} \geq n_{1}$ and $\lambda \in(0,1]$,

$$
\lambda a_{\bar{n}_{0}} \notin\left\{a_{n}\right\},
$$

then we can take some $t_{n}$ from $\left\{t_{n}\right\}$ such that $\frac{t_{\bar{n}_{0}}}{t_{n}}<1$ as $n \geq \bar{n}_{0}$. From (2.6) it follows that $\frac{t_{\bar{n}_{0}}}{t_{n}} a_{\bar{n}_{0}} \notin\left\{a_{n}\right\}$ as $n \geq \bar{n}_{0}$. Thus $\left\|t_{n}\left(\frac{t_{\bar{n}_{0}}}{t_{n}} a_{\bar{n}_{0}}\right)-u\right\|=\left\|t_{\bar{n}_{0}} a_{\bar{n}_{0}}-u\right\|>\varepsilon_{1}$, a contradiction. So (2.5) holds. Again from $t_{n} a_{n} \rightarrow u$, so, $t_{n_{m}} a_{n_{m}}=t_{n_{m}} \lambda_{n_{m}} a_{\bar{n}_{0}} \rightarrow u$. Furthermore, $a_{\bar{n}_{0}}$ is fixed, so $t_{n_{m}} \lambda_{n_{m}} \rightarrow t_{0}$. That is, $t_{n_{m}} \lambda_{n_{m}}=t_{0}+\bar{t}_{n_{m}}$ with $\bar{t}_{n_{m}} \rightarrow 0$. Thus, $t_{n_{m}} a_{n_{m}}=\left(t_{0}+\bar{t}_{n_{m}}\right) a_{\bar{n}_{0}}=t_{0} a_{\bar{n}_{0}}+$ $\bar{t}_{n_{m}} a_{\bar{n}_{0}} \rightarrow t_{0} a_{\bar{n}_{0}}$. From the uniqueness of limits, we have $u=t_{0} a_{\bar{n}_{0}}$ with $a_{\bar{n}_{0}} \in \operatorname{cl} A \backslash \operatorname{int} A$, so $u \in \operatorname{cone}(\operatorname{cl} A)$.

(ii) If $\left\{a_{n}\right\} \subset \operatorname{int} A$, then for any $n, \exists \varepsilon_{n}$ such that $B\left(a_{n}, \varepsilon_{n}\right) \subset A$. From $t_{n} a_{n}=u+x_{n}$, one has $a_{n}-\frac{u}{t_{n}}=\frac{x_{n}}{t_{n}}$ and $a_{n} \in B\left(0_{X}, \varepsilon\right)$ as $n$ is large enough. If there exists $n_{0}$ such that $\| a_{n_{0}}-$ $\frac{u}{t_{n_{0}}} \|=\frac{\left\|x_{n_{0}}\right\|}{t_{n_{0}}} \leq \varepsilon_{n_{0}}$, then $\frac{u}{t_{n_{0}}} \in A$, so $u \in \operatorname{cone}(\operatorname{cl} A)$. If, for any $n,\left\|a_{n}-\frac{u}{t_{n}}\right\|=\left\|\frac{x_{n}}{t_{n}}\right\|>\varepsilon_{n}$, then this implies there exist $\bar{a}_{n} \in \operatorname{cl} A \backslash A$ and $\left\|\bar{a}_{n}-a_{n}\right\| \leq \frac{\left\|x_{n}\right\|}{t_{n}}$. However, $\left\|t_{n} \bar{a}_{n}-u\right\|=$ $\left\|t_{n} \bar{a}_{n}-t_{n} a_{n}+t_{n} a_{n}-u\right\| \leq t_{n} \frac{\left\|x_{n}\right\|}{t_{n}}+\left\|x_{n}\right\|=2\left\|x_{n}\right\| \rightarrow 0$. Thus, $t_{n} \bar{a}_{n} \rightarrow u$. By $\bar{a}_{n} \in \operatorname{cl} A \backslash A$ and (i), we conclude that $u \in \operatorname{cone}(\operatorname{cl} A)$.

On the other hand, if $a_{n} \nrightarrow 0_{X}$, then $\left\{t_{n}\right\}$ is bounded. Set $t=\sup _{n}\left\{t_{n}\right\}$, then $t \geq t_{n}$ and $t \in$ $R^{+}$. Dividing $t$ by (2.4) and for each $n \in N$, set $b_{n}=\frac{t_{n} a_{n}}{t}=\frac{u}{t}+\frac{x_{n}}{t}$. According to assumption $A$ satisfying the property $\digamma$, we get $b_{n} \in A$ and $b_{n} \rightarrow \frac{u}{t}$ as $n \rightarrow \infty$. Thus, $\frac{u}{t} \in \operatorname{cl} A$, i.e., $u \in \operatorname{cone}(\mathrm{cl} A)$. From the above two parts, we get cone $(\mathrm{cl} A)$ is a closed cone, $\operatorname{socl}(\operatorname{cone} A) \subseteq$ $\operatorname{cone}(\operatorname{cl} A)$. The proof is complete.

Corollary 2 Let $A$ be a cone of $X$ and satisfy the property $\Lambda$ near the $0_{X}$, then $\operatorname{cone}(\operatorname{cl} A)=$ $\operatorname{cl} A$.

Corollary 3 Let $x_{0} \in A$, if $A$ is a closed convex subset of $X$ such that $A-x_{0}$ satisfy the property $\Lambda$ near the $0_{X}$, then cone $\left(A-x_{0}\right)=\operatorname{clcone}\left(A-x_{0}\right)$.

Proposition 2.4 (see Chapter 4 of [3]) Let $x_{0} \in A \subset X$, if $A$ is star-shaped at $x_{0}$, then $T\left(A, x_{0}\right)=\operatorname{cl}\left(\operatorname{cone}\left(A-x_{0}\right)\right)$.

Corollary 4 Let $x_{0} \in A \subset X$, if $A$ is a closed convex subset such that $A-x_{0}$ satisfy the property $\Lambda$ near the $0_{X}$, then $T\left(A, x_{0}\right)=\operatorname{cone}\left(A-x_{0}\right)$.

Proof Since $x_{0} \in A$ and $A$ is a convex subset, we have $T\left(A, x_{0}\right)=\operatorname{cl}\left(\operatorname{cone}\left(A-x_{0}\right)\right)$. To apply Corollary 3 , one has

$$
\operatorname{cl}\left(\operatorname{cone}\left(A-x_{0}\right)\right)=\operatorname{cone}\left(A-x_{0}\right)
$$

it is clear that $T\left(A, x_{0}\right)=\operatorname{cone}\left(A-x_{0}\right)$. 


\section{The existence theory for contingent epiderivative}

The aim of this section is to discuss the existence of the contingent epiderivative for a set-valued map defined from a real normed space to a real normed space. Let $A \subset X$, $F: A \rightarrow 2^{Y}$ be a set-valued mapping.

Definition 3.1 (see [1]) Let $x_{0} \in A$ and a pair $\left(x_{0}, y_{0}\right) \in \operatorname{gr} F$ be given. The contingent epiderivative $D F\left(x_{0}, y_{0}\right)$ of $F$ at $\left(x_{0}, y_{0}\right)$ is the single-valued mapping from $X$ to $Y$ defined by

$$
\operatorname{epi}\left(D F\left(x_{0}, y_{0}\right)\right)=T\left(\operatorname{epi} F,\left(x_{0}, y_{0}\right)\right)
$$

Next, we establish $D F\left(x_{0}, y_{0}\right)$ in the following (3.6) and also prove it is a single-valued mapping.

Let a pair $\left(x_{0}, y_{0}\right) \in \operatorname{gr} F$, for any $x \in X$, set

$$
D F\left(x_{0}, y_{0}\right)(x)=\operatorname{IMin}\left\{y \mid(x, y) \in T\left(\text { epi } F,\left(x_{0}, y_{0}\right)\right)\right\} .
$$

Notice that if $A \subset X$ is a closed convex set, $F$ is $D$-convex and epi $F$ is a closed subset in $X \times Y$ such that (epi $\left.F-\left(x_{0}, y_{0}\right)\right)$ satisfies the property $\Lambda$ near the $\left(0_{X}, 0_{Y}\right)$, then

$$
\begin{aligned}
T\left(\text { epi } F,\left(x_{0}, y_{0}\right)\right) & =\operatorname{cone}\left(\operatorname{epi} F-\left\{\left(x_{0}, y_{0}\right)\right\}\right) \\
& =\operatorname{cone}\left((\operatorname{gr} F+\{0\} \times D)-\left\{\left(x_{0}, y_{0}\right)\right\}\right) \\
& =\operatorname{cone}\left((A \times F(A)+\{0\} \times D)-\left\{\left(x_{0}, y_{0}\right)\right\}\right) \\
& =\bigcup_{a \in A} \operatorname{cone}\left((\{a\} \times F(a)+\{0\} \times D)-\left\{\left(x_{0}, y_{0}\right)\right\}\right),
\end{aligned}
$$

is a closed convex cone. So, for every $(x, y) \in \operatorname{epi}\left(D F\left(x_{0}, y_{0}\right)\right)$ and $(x, y) \neq\left(0_{X}, 0_{Y}\right)$, if $(x, y) \in$ $T\left(\right.$ epi $\left.F,\left(x_{0}, y_{0}\right)\right)$, then there exist $l>0, a \in A, v \in F(a)$ and $d \in D$ such that

$$
(x, y)=l\left(a-x_{0}, v-y_{0}+d\right)
$$

By (3.3), we have

$$
x=l\left(a-x_{0}\right) \in \operatorname{cone}\left(A-x_{0}\right),
$$

and

$$
y=l\left(v-y_{0}+d\right) \in \operatorname{cone}\left(F\left(x_{0}+\lambda x\right)-y_{0}+D\right)
$$

where $\lambda=\frac{1}{l}$.

Otherwise, if $(x, y)=\left(0_{X}, 0_{Y}\right)$, then we can conclude that $a=x_{0}, v=y_{0}$ and $d=y_{0}$. Therefore, formulas (3.4) and (3.5) are clearly established.

Remark 3.1 From the above discussion and Corollary 4, if $A \subset X$ is a closed convex set and $A-x_{0}$ satisfies the property $\Lambda$ near the $0_{X}, F: A \rightarrow 2^{Y}$ is $D$-convex and epi $F$ is closed in $X \times Y$ such that (epi $\left.F-\left(x_{0}, y_{0}\right)\right)$ satisfies the property $\Lambda$ near the $\left(0_{X}, 0_{Y}\right)$, then $D F\left(x_{0}, y_{0}\right)$ can be defined on $T\left(A, x_{0}\right)$. 
Furthermore, we can conclude that formula (3.2) is equivalent to the following:

$$
D F\left(x_{0}, y_{0}\right)(x)=\operatorname{IMin}\left(\operatorname{cone}\left(F\left(x_{0}+\lambda x\right)-y_{0}+D\right)\right),
$$

where $x \in T\left(A, x_{0}\right)$ and $\lambda \geq 0$.

From Theorem 2.1, we can obtain the following existence theorem.

Theorem 3.1 Let $A \subset X$ be a closed convex set, let $F: A \rightarrow 2^{Y}$ be $D$-convex, and let epi $F$ be a closed subset in $X \times Y$. Let $\left(x_{0}, y_{0}\right) \in \operatorname{gr} F$. If for any $x \in T\left(A, x_{0}\right)$ and $\lambda \geq 0$, the following conditions hold: (1) $\left(F\left(x_{0}+\lambda x\right)-y_{0}+D\right)$ satisfies the properties $\digamma$ and $\Lambda$ near the $0_{Y}$; (2) cone $\left(F\left(x_{0}+\lambda x\right)-y_{0}+D\right)$ is D-lower bounded; (3) the cone D is Daniell cone; (4) $\operatorname{cone}\left(F\left(x_{0}+\lambda x\right)-y_{0}+D\right)$ satisfies (2.1). Then IMin $\left(\operatorname{cone}\left(F\left(x_{0}+\lambda x\right)-y_{0}+D\right)\right) \neq \emptyset$ is a single-point set.

Proof Since epi $F$ a closed convex subset in $X \times Y$, by Proposition 2.1, one has $F\left(x_{0}+\right.$ $\lambda x)+D$ a closed set for each $x \in T\left(A, x_{0}\right)$, so is $\left(F\left(x_{0}+\lambda x\right)-y_{0}+D\right)$. By assumption (1) and Proposition 2.3, we can conclude that cone $\left(F\left(x_{0}+\lambda x\right)-y_{0}+D\right)$ is a closed set. Considering Theorem 2.1 and given conditions (2) and (3), we have $\operatorname{Min}\left(\operatorname{cone}\left(F\left(x_{0}+\lambda x\right)-y_{0}+D\right)\right) \neq \emptyset$. Combining Remark 2.1 and assumption (4), we can conclude IMin(cone $\left(F\left(x_{0}+\lambda x\right)-y_{0}+\right.$ $D)) \neq \emptyset$ is a single-point set.

Remark 3.2 From (3.6), we know that $D F\left(x_{0}, y_{0}\right)$ exists if $\operatorname{IMin}\left(\operatorname{cone}\left(F\left(x_{0}+\lambda x\right)-y_{0}+\right.\right.$ $D)) \neq \emptyset$.

The following theorem shows that our definition of the contingent epiderivative for a set-valued mapping is well defined.

Theorem 3.2 If all the conditions of Theorem 3.1 are fulfilled, then epi $\left(D F\left(x_{0}, y_{0}\right)\right)=$ $T\left(\right.$ epi $\left.F,\left(x_{0}, y_{0}\right)\right)$.

Proof Combining (3.6) and Theorem 3.1, we get $D F\left(x_{0}, y_{0}\right)(x)$ exists for each $x \in T\left(A, x_{0}\right)$. Since $\left(x, D F\left(x_{0}, y_{0}\right)(x)\right) \in$ cone $\left(\right.$ epi $\left.F-\left(x_{0}, y_{0}\right)\right)$, thus

$$
\begin{aligned}
\operatorname{epi}\left(D F\left(x_{0}, y_{0}\right)\right) & \subset \operatorname{cone}\left(\text { epi } F-\left\{\left(x_{0}, y_{0}\right)\right\}\right)+\{0\} \times D \\
& =\text { cone }\left(\text { epi } F-\left\{\left(x_{0}, y_{0}\right)\right\}\right) \\
& =T\left(\text { epi } F,\left(x_{0}, y_{0}\right)\right) .
\end{aligned}
$$

Now, let us show the converse inclusion. From the definition of the $D F\left(x_{0}, y_{0}\right)$, for any $(x, y) \in T\left(\right.$ epi $\left.F,\left(x_{0}, y_{0}\right)\right)$, we have

$$
\begin{aligned}
(x, y) & \in\left(\left(x, D F\left(x_{0}, y_{0}\right)\right)+\{0\} \times D\right) \\
& \subset \operatorname{gr}\left(D F\left(x_{0}, y_{0}\right)\right)+\left\{0_{Y}\right\} \times D \\
& =\operatorname{epi}\left(D F\left(x_{0}, y_{0}\right)\right) .
\end{aligned}
$$

Therefore,

$$
T\left(\text { epi } F,\left(x_{0}, y_{0}\right)\right) \subset \operatorname{epi}\left(D F\left(x_{0}, y_{0}\right)\right) .
$$

The proof is complete. 
Next we show under appropriate assumptions that the contingent epiderivative is a strictly positive homogeneous and subadditive map in the case of $A$, a convex set, and epi $F$, a closed convex subset.

Definition 3.2 Let $X$ be a real linear space and let $Y$ be a real linear space partially ordered by a closed convex pointed cone $D \subset Y$. A map $f: X \rightarrow 2^{Y}$ is called

(a) strictly positive homogeneous if

$$
f(\alpha x)=\alpha f(x) \quad \text { for all } \alpha>0 \text { and all } x \in X
$$

(b) subadditive if

$$
f\left(x_{1}\right)+f\left(x_{2}\right) \subset f\left(x_{1}+x_{2}\right)+D \text { for all } x_{1}, x_{2} \in X
$$

If the properties under (a) with $\alpha \geq 0$ and (b) hold, then $f$ is called sublinear.

Theorem 3.3 Let $A$ be a closed convex set in $X, x_{0} \in A$ such that $A-x_{0}$ satisfies the property $\Lambda$ near the $0_{X}$. Let $D$ be a closed convex pointed cone in $Y$ and $\left(x_{0}, y_{0}\right) \in \operatorname{gr} F$ such that epi $F$ is a closed convex set and (epi $\left.F-\left(x_{0}, y_{0}\right)\right)$ satisfies the property $\Lambda$ near the $\left(0_{X}, 0_{Y}\right)$. If for all $x \in T\left(A, x_{0}\right), D F\left(x_{0}, y_{0}\right)(x)$ exists, then $D F\left(x_{0}, y_{0}\right)(x)$ is sublinear.

Proof We take any $\alpha>0$ and any $x \in T\left(A, x_{0}\right)$. Then we obtain

$$
\begin{aligned}
D F\left(x_{0}, y_{0}\right)(\alpha x) & =\operatorname{IMin}\left\{y \mid(\alpha x, y) \in T\left(\operatorname{epi} F,\left(x_{0}, y_{0}\right)\right)\right\} \\
& =\operatorname{IMin}\left\{y \mid y \in\left(\operatorname{cone}\left(F\left(x_{0}+\lambda \alpha x\right)-y_{0}+D\right)\right)\right\} \\
& =\operatorname{IMin}\left\{\alpha v \mid \alpha v \in\left(\operatorname{cone}\left(F\left(x_{0}+\lambda \alpha x\right)-y_{0}+D\right)\right)\right\} \\
& =\alpha \operatorname{IMin}\left\{v \mid v \in\left(\operatorname{cone}\left(F\left(x_{0}+\lambda x\right)-y_{0}+D\right)\right)\right\} .
\end{aligned}
$$

Thus $D F\left(x_{0}, y_{0}\right)$ is strictly positive homogeneous.

Next, for $x_{1}, x_{2} \in T\left(A, x_{0}\right)$, we have $\left(x_{1}, D F\left(x_{0}, y_{0}\right)\left(x_{1}\right)\right) \in T\left(\right.$ epi $\left.F,\left(x_{0}, y_{0}\right)\right)$ and $\left(x_{2}, D F\left(x_{0}\right.\right.$, $\left.\left.y_{0}\right)\left(x_{2}\right)\right) \in T\left(\right.$ epi $\left.F,\left(x_{0}, y_{0}\right)\right)$. Since $F$ is $D$-convex and epi $F$ is a closed subset, then $T$ (epi $F$, $\left.\left(x_{0}, y_{0}\right)\right)$ is a closed convex cone. Thus,

$$
\left(x_{1}+x_{2}, D F\left(x_{0}, y_{0}\right)\left(x_{1}\right)+D F\left(x_{0}, y_{0}\right)\left(x_{2}\right)\right) \in T\left(\text { epi } F,\left(x_{0}, y_{0}\right)\right) \text {, }
$$

implies

$$
D F\left(x_{0}, y_{0}\right)\left(x_{1}\right)+D F\left(x_{0}, y_{0}\right)\left(x_{2}\right) \in \operatorname{cone}\left(F\left(x_{0}+\lambda\left(x_{1}+x_{2}\right)\right)-y_{0}+D\right) \text {. }
$$

By Remark 2.1, we have

$$
\begin{aligned}
D F\left(x_{0}, y_{0}\right)\left(x_{1}\right)+D F\left(x_{0}, y_{0}\right)\left(x_{2}\right) & \in \operatorname{cone}\left(F\left(x_{0}+\lambda\left(x_{1}+x_{2}\right)\right)-y_{0}+D\right) \\
& \subset \operatorname{IMin}\left(\operatorname{cone}\left(F\left(x_{0}+\lambda\left(x_{1}+x_{2}\right)\right)-y_{0}+D\right)\right)+D \\
& =D F\left(x_{0}, y_{0}\right)\left(x_{1}+x_{2}\right)+D .
\end{aligned}
$$

The proof is complete. 


\section{Relationships between vector variational-like inequalities and optimization problems}

In this section, we restrict ourselves to dealing with relationships between two kinds of vector variational-like inequality problems and set-valued optimization problems.

The set-valued vector optimization problem under our consideration is

$$
\operatorname{Min}\{F(x): x \in A\}
$$

where $F: X \rightarrow 2^{Y}, F(A)=\bigcup_{x \in A} F(x)$. We denote set-valued optimization problem (4.1) as (SOP).

Definition 4.1 Consider the above problem (SOP), let $x_{0} \in A, y_{0} \in F\left(x_{0}\right)$.

(i) A pair $\left(x_{0}, y_{0}\right) \in \operatorname{gr} F$ is called a minimal solution of $F$ on $A$ if $\left(F(A)-y_{0}\right) \cap-D=\left\{0_{Y}\right\}$;

(ii) A pair $\left(x_{0}, y_{0}\right) \in \operatorname{gr} F$ is called an ideal minimal solution of $F$ on $A$ if $\left(F(A)-y_{0}\right) \subseteq D$.

The sets of all minimal solutions and ideal minimal solutions of (SOP) are denoted by $\operatorname{Min}(F, A)$ and $\operatorname{IMin}(F, A)$, respectively.

In the following, we always assume that contingent epiderivative of $F$ exists.

Definition 4.2 (see [14]) Let the set $A$ be convex, let the set-valued mapping $F: A \rightarrow 2^{Y}$ be $D$-convex. Let $\left(x_{0}, y_{0}\right) \in \operatorname{gr} F$ and let the contingent epiderivative $D F\left(x_{0}, y_{0}\right)$ of $F$ at $\left(x_{0}, y_{0}\right)$ exist.

(i) A linear map $L: X \rightarrow Y$, with $L(x) \leq D F\left(x_{0}, y_{0}\right)(x)$, for all $x \in T\left(A, x_{0}\right)$ is called a subgradient of $F$ at $\left(x_{0}, y_{0}\right)$.

(ii) The set $\partial F\left(x_{0}, y_{0}\right)=\left\{L: X \rightarrow Y: L(x) \leq D F\left(x_{0}, y_{0}\right)(x), \forall x \in T\left(A, x_{0}\right)\right\}$ of all subgradients $L$ of $F$ at $\left(x_{0}, y_{0}\right)$ is called the subdifferential of $F$ at $\left(x_{0}, y_{0}\right)$.

Definition 4.3 A set $A \subset X$ is said to be an invex set if there exists a function $\eta: X \times X \rightarrow$ $X$ such that $\forall x, y \in A, \forall \lambda \in[0,1], y+\lambda \eta(x, y) \in A$.

Throughout the paper, we always assume that $A$ is an invex subset of $X$, the function $\eta$ defined on $A$, i.e., $\eta: A \times A \rightarrow X$ and the subdifferential of $F$ exists at every $(x, y) \in \operatorname{gr} F$.

Definition 4.4 Let $F: A \rightarrow 2^{Y}$ be a set-valued mapping, $\left(x_{0}, y_{0}\right) \in \operatorname{gr} F$. $F$ is called strong pseudo-invex at $\left(x_{0}, y_{0}\right)$ with respect to $\eta$ on $A$ if $\forall x \in A, \forall y \in F(x)$ and $\forall L \in \partial F\left(x_{0}, y_{0}\right)$, $y-y_{0} \notin D \Rightarrow L \eta\left(x, x_{0}\right) \notin D$.

$F$ is said to be strong pseudo-invex with respect to $\eta$ on $A$ if for every pair $(x, y) \in \operatorname{gr} F$, $F$ is strong pseudo-invex at $(x, y)$ with respect to $\eta$.

Definition 4.5 Let $F: A \rightarrow 2^{Y}$ be a set-valued mapping, $\left(x_{0}, y_{0}\right) \in \operatorname{gr} F$. $F$ is called pseudoinvex at $\left(x_{0}, y_{0}\right)$ with respect to $\eta$ on $A$ if $\forall x \in A, \forall y \in F(x)$ and $\forall L \in \partial F\left(x_{0}, y_{0}\right), y-y_{0} \in$ $-D \Rightarrow L \eta\left(x, x_{0}\right) \in-D$.

$F$ is said to be pseudo-invex with respect to $\eta$ on $A$ if for every pair $(x, y) \in \operatorname{gr} F, F$ is pseudo-invex at $(x, y)$ with respect to $\eta$.

Definition 4.6 Let $F: A \rightarrow 2^{Y}$ be a set-valued mapping. $F$ is said to be $D$-preinvex with respect to $\eta$ on $A$ if $\forall x, y \in A, \forall \lambda \in[0,1], \lambda F(x)+(1-\lambda) F(y) \subset F(y+\lambda \eta(x, y))+D$. 
From the above definitions, we have the following useful proposition.

Proposition 4.1 Let $F: A \rightarrow 2^{Y}$ be D-preinvex with respect to $\eta$ on $A,\left(x_{0}, y_{0}\right) \in \operatorname{gr} F$. If $D F\left(x_{0}, y_{0}\right)$ exists on cone $\left(A-x_{0}\right)$, then for all $L \in \partial F\left(x_{0}, y_{0}\right)$, all $x \in A$, all $y \in F(x), y-y_{0} \in$ $\left\langle L, \eta\left(x, x_{0}\right)\right\rangle+D$.

Proof For all $x \in A$, since $F$ is $D$-preinvex with respect to $\eta$ on $A$ and $x_{0} \in A$, for any $\lambda \in$ $[0,1]$, we obtain

$$
(1-\lambda) F\left(x_{0}\right)+\lambda F(x) \subset F\left(x_{0}+\lambda \eta\left(x, x_{0}\right)\right)+D,
$$

which implies that

$$
y-y_{0} \in\left(F\left(x_{0}+\lambda \eta\left(x, x_{0}\right)\right)+D-y_{0}\right) / \lambda .
$$

Thus, we get

$$
y-y_{0} \in D F\left(x_{0}, y_{0}\right)\left(\eta\left(x, x_{0}\right)\right)+D
$$

It is clear that

$$
\forall x \in A, \quad \eta\left(x, x_{0}\right) \in \operatorname{dom} D F\left(x_{0}, y_{0}\right) .
$$

By the definition of subdifferential $\partial F\left(x_{0}, y_{0}\right)$, for all $L \in \partial F\left(x_{0}, y_{0}\right)$, we have

$$
L(x) \leq D F\left(x_{0}, y_{0}\right)(x), \quad \forall x \in \operatorname{dom} D F\left(x_{0}, y_{0}\right),
$$

that is,

$$
D F\left(x_{0}, y_{0}\right)(x) \in L(x)+D .
$$

Combining (4.3), (4.4) and (4.5), we can conclude, $y-y_{0} \in\left\langle L, \eta\left(x, x_{0}\right)\right\rangle+D$.

Remark 4.1 It is easy to see that $F$ is pseudo-invex with respect to $\eta$ on $A$ if $F$ is $D$-preinvex with respect to $\eta$ on $A$.

A vector variational inequality has been shown to be a useful tool in vector optimization. Some authors have proved the equivalence between them, see $[9,10,15]$. The vector variational-like inequality problem is a generalized form of the vector variational inequality problem, which was introduced and studied by [8].

Now, let us offer the following definitions.

A vector variational-like inequality problem (VVLI) is to find $\left(x_{0}, y_{0}\right) \in \operatorname{gr} F$ such that $\left\langle L, \eta\left(x, x_{0}\right)\right\rangle \notin-D \backslash\left\{0_{Y}\right\}$ for all $x \in A \backslash\left\{x_{0}\right\}$ and all $L \in \partial F\left(x_{0}, y_{0}\right)$. A strong vector variational-like inequality problem (SVVLI) is to find $\left(x_{0}, y_{0}\right) \in \operatorname{gr} F$ such that $\left\langle L, \eta\left(x, x_{0}\right)\right\rangle \in$ $D$ for all $x \in A$ and all $L \in \partial F\left(x_{0}, y_{0}\right)$.

In the following, using the tools of non-smooth analysis and the concept of non-smooth (strong) vector pseudo-invexity, we shall obtain the stronger results than those of [11]. 
Theorem 4.1 Let $x_{0} \in A,\left(x_{0}, y_{0}\right) \in \operatorname{gr} F$. If the pair $\left(x_{0}, y_{0}\right)$ solves the (SVVIL) and $F$ is strong pseudo-invex at $\left(x_{0}, y_{0}\right)$ with respect to $\eta$ on $A$, then $\left(x_{0}, y_{0}\right)$ is an ideal minimizer of the $(S O P)$.

Proof Since the pair $\left(x_{0}, y_{0}\right)$ solves the (SVVIL), we have that

$$
\left\langle L, \eta\left(x, x_{0}\right)\right\rangle \in D, \quad \forall x \in A, \forall L \in \partial F\left(x_{0}, y_{0}\right) .
$$

If $\left(x_{0}, y_{0}\right)$ is not an ideal minimizer of the (SOP), then there exist $\bar{x} \in A,(\bar{x}, \bar{y}) \in \operatorname{gr} F$ such that

$$
\bar{y}-y_{0} \notin D .
$$

Since $F$ is strong pseudo-invex at $\left(x_{0}, y_{0}\right)$ with respect to $\eta$ on $A$, we get $\bar{x} \in A, \forall L \in$ $\partial F\left(x_{0}, y_{0}\right)$ such that

$$
\bar{y}-y_{0} \notin D \Rightarrow\left\langle L, \eta\left(\bar{x}, x_{0}\right)\right\rangle \notin D .
$$

By (4.7) and (4.8), we obtain $\left\langle L, \eta\left(\bar{x}, x_{0}\right)\right\rangle \notin D$, which contradicts (4.6).

In order to see the converse of the above theorem, we must impose stronger conditions, as can be observed in the following theorem.

Theorem 4.2 Suppose that $F: A \rightarrow 2^{Y}$ and $-F$ is D-preinvex with respect to $\eta$ on $A$, $\left(x_{0}, y_{0}\right) \in \operatorname{gr} F$. If $-\partial F\left(x_{0}, y_{0}\right) \subseteq \partial(-F)\left(x_{0},-y_{0}\right)$ and the pair $\left(x_{0}, y_{0}\right)$ is an ideal minimizer of $(S O P)$, then $\left(x_{0}, y_{0}\right)$ solves the (SVVLI).

Proof Let $\left(x_{0}, y_{0}\right)$ be an ideal minimizer of the (SOP), then we have

$$
y-y_{0} \in D, \quad \forall x \in A, \forall y \in F(x) .
$$

Since $-F$ is $D$-preinvex with respect to $\eta$ and $-\partial F\left(x_{0}, y_{0}\right) \subseteq \partial(-F)\left(x_{0},-y_{0}\right)$, we have that $\forall x \in A, \forall y \in F(x)$ and $\forall L \in \partial F\left(x_{0}, y_{0}\right)$ such that

$$
-y+y_{0} \in\left\langle-L, \eta\left(x, x_{0}\right)\right\rangle+D,
$$

which implies

$$
\left\langle L, \eta\left(x, x_{0}\right)\right\rangle \in y-y_{0}+D
$$

Combining (4.9) and (4.10), we can conclude

$$
\left\langle L, \eta\left(x, x_{0}\right)\right\rangle \in D+D \subset D
$$

for all $x \in A$ and all $L \in \partial F\left(x_{0}, y_{0}\right)$. That is, $\left(x_{0}, y_{0}\right)$ solves the (SVVLI). 
Theorem 4.3 Suppose that $F: A \rightarrow 2^{Y}$ and $-F$ is D-preinvex with respect to $\eta$ on $A$, $\left(x_{0}, y_{0}\right) \in \operatorname{gr} F$.If $-\partial F\left(x_{0}, y_{0}\right) \subseteq \partial(-F)\left(x_{0},-y_{0}\right)$ and the pair $\left(x_{0}, y_{0}\right)$ is a minimizer of $(S O P)$, then $\left(x_{0}, y_{0}\right)$ solves the $(V V L I)$.

Proof Let $\left(x_{0}, y_{0}\right)$ be a minimizer of the (SOP), then we have

$$
y-y_{0} \notin\left(-D \backslash\left\{0_{Y}\right\}\right), \quad \forall x \in A \backslash\left\{x_{0}\right\}, \forall y \in F(x) .
$$

If $\left(x_{0}, y_{0}\right)$ does not solve the (VVIL), then there exist $\bar{x} \in A \backslash\left\{x_{0}\right\}$ and $\bar{L} \in \partial F\left(x_{0}, y_{0}\right)$ such that

$$
\left\langle\bar{L}, \eta\left(\bar{x}, x_{0}\right)\right\rangle \in\left(-D \backslash\left\{0_{Y}\right\}\right) .
$$

Since $-F$ is $D$-preinvex with respect to $\eta$ and $-\partial F\left(x_{0}, y_{0}\right) \subseteq \partial(-F)\left(x_{0},-y_{0}\right)$, we have that $\forall x \in A, \forall y \in F(x)$ and $\bar{L} \in \partial F\left(x_{0}, y_{0}\right)$ such that

$$
-y+y_{0} \in\left\langle-\bar{L}, \eta\left(x, x_{0}\right)\right\rangle+D .
$$

Combining (4.12) and (4.13), for all $\bar{y} \in F(\bar{x})$, one has

$$
-\bar{y}+y_{0} \in D \backslash\left\{0_{Y}\right\}+D \subset D \backslash\left\{0_{Y}\right\},
$$

which contradicts (4.11). The proof is complete.

The converse case of the above theorem (see Theorem 4.4 of [11]) only requires $F$ to be pseudo-invex.

\section{Competing interests}

The authors declare that they have no competing interests.

\section{Authors' contributions}

YC carried out the main preliminaries, the existence theory for contingent epiderivative and the relationships between vector variational-like inequalities and optimization problems. SL carried out the introduction and some preliminaries. YL participated in the design of the study and some preliminaries.

\section{Author details}

'Department of Mathematics, Xidian University, Xi'an, 710071, China. ${ }^{2}$ Baoji University of Arts and Science, Baoji, 721016, China.

\section{Acknowledgements}

This work was supported by the Fundamental Research Funds for the Central Universities, No. K5051370004.

Received: 17 September 2012 Accepted: 12 July 2013 Published: 29 July 2013

\section{References}

1. Jahn, J, Raüh, R: Contingent epiderivatives and set-valued optimization. Math. Methods Oper. Res. 46, $193-211$ (1997)

2. Aubin, JP, Frankowska, H: Set-Valued Analysis. Birkhäuser, Basel (1990)

3. Jahn, J, Khan, AA: The existence of contingent epiderivatives for set-valued maps. Appl. Math. Lett. 16, 1179-1185 (2003)

4. Rodríguez-Marín, L, Sama, M: About contingent epiderivatives. J. Math. Anal. Appl. 327, 745-762 (2007)

5. Lalitha, CS, Arora, R: Weak Clarke epiderivative in set-valued optimization. J. Math. Anal. Appl. 342, 704-714 (2008)

6. Chen, GY, Jahn, J: Optimality conditions for set-valued optimization problems. Math. Methods Oper. Res. 48, 187-200 (1998)

7. Rodríguez-Marín, L, Sama, M: Variational characterization of the contingent epiderivative. J. Math. Anal. Appl. 335 1374-1382 (2007)

8. Ruiz-Garzón, G, Osuna-Gómez, R, Rufián-Lizana, A: Relationships between vector variational-like inequality and optimization problems. Eur. J. Oper. Res. 157, 113-119 (2004) 
9. Mishra, SK, Wang, SY: Vector variational-like inequalities and non-smooth vector optimization problems. Nonlinear Anal. 64, 1939-1945 (2006)

10. Rezaie, M, Zafarani, J: Vector optimization and variational-like inequalities. J. Glob. Optim. 43, 47-66 (2009)

11. Zeng, J, Li, SJ: On vector variational-like inequalities and set-valued optimization problems. Optim. Lett. 5, 55-69 (2011)

12. Hamel, AH: A duality theory for set-valued functions I: Fenchel conjugation theory. Set-Valued Anal. 17, 153-182 (2009)

13. Borwein, JM: On the existence of Pareto efficient points. Math. Oper. Res. 8, 64-73 (1983)

14. Baier, J, Jahn, J: Technical note on subdifferentials of set-valued maps. J. Optim. Theory Appl. 100, $233-240$ (1999)

15. Lee, GM, Kim, DS, Lee, BS, Cho, SJ: On vector variational inequality. Bull. Korean Math. Soc. 33(4), 553-564 (1996)

doi:10.1186/1029-242X-2013-352

Cite this article as: Chai et al.: The existence of contingent epiderivative for a set-valued mapping and vector variational-like inequalities. Journal of Inequalities and Applications 2013 2013:352.

Submit your manuscript to a SpringerOpen ${ }^{\odot}$ journal and benefit from:

- Convenient online submission

- Rigorous peer review

- Immediate publication on acceptance

- Open access: articles freely available online

- High visibility within the field

- Retaining the copyright to your article 